\title{
Skeletal muscle dysfunction: a ubiquitous outcome in chronic disease?
}

\author{
James Nathan, ${ }^{1}$ Jonathan Fuld ${ }^{2}$
}

\begin{abstract}
Skeletal muscle wasting is a debilitating consequence of respiratory disease, particularly well characterised in individuals with chronic obstructive pulmonary disease (COPD). However, it remains controversial whether peripheral muscle dysfunction is an intrinsic part of the respiratory disease process, or a generalised response to multiple atrophy stimuli. Such debate has not only informed the subtleties of pathophysiology, but has also instigated detailed analyses of the molecular mechanisms involved in the induction of muscle atrophy, identifying the ubiquitin-proteasome system (UPS) as the predominant pathway required for the rapid proteolysis seen in muscle wasting.
\end{abstract}

In this issue of Thorax (see page 113), Mainguy et al contribute to our knowledge of muscle weakness in chronic respiratory disease, characterising peripheral muscle function in patients with idiopathic pulmonary arterial hypertension (IPAH). ${ }^{1}$ Ten WHO functional class II-III patients with IPAH and 10 matched healthy controls underwent exercise capacity assessment, quadriceps strength testing and peripheral muscle morphology analysis. Patients with IPAH demonstrated a lower proportion of type I fibres in vastus lateralis muscle biopsies than healthy controls (38\% vs 50\%), and this was associated with reduced quadriceps strength and a relatively higher potential for anaerobic metabolism (enzymatic ratio of phosphofructokinase (PFK)/3-hydroxyacyl CoA dehydrogenase (HDAH)). While there was no correlation between the proportion of type I fibres and muscle strength with pulmonary haemodynamic parameters, a positive correlation was demonstrated for peripheral muscle strength and exercise capacity $\left(R^{2}=0.42, \mathrm{p}=0.04\right)$. The authors conclude that peripheral muscle dysfunction may contribute to exercise intolerance

${ }^{1}$ University of Cambridge, Cambridge, UK ${ }^{2}$ Addenbroke's Hospital, Cambridge, UK

Correspondence to Dr Jonathan Fuld, Addenbroke's Hosital, 1B Humberstone Road, Cambridge CB4 1JD, UK; jonathanfuld@gmail.com in patients with IPAH. With relatively small numbers in the study groups, the association between peripheral muscle strength and exercise capacity should be interpreted with caution. No difference in muscle strength was detected when corrected for muscle cross-sectional area and, apart from the proportion of type I fibres, no other differences in muscle morphology were identified between the groups. It is therefore uncertain that the changes in enzymatic ratios correlate directly with fibre type. Despite these limitations, this study prompts the elucidation of molecular mechanisms involved in peripheral muscle dysfunction, and invites a formalised investigation into the impact of exercise training upon physiological and clinical measures in IPAH.

The findings of Mainguy et al are consistent with previous studies examining muscle dysfunction in IPAH. Both isometric forearm muscle strength and respiratory mouth pressures are observed to reduce in patients with IPAH. $^{2}{ }^{3}$ Peripheral muscle strength has also been shown to correlate with 6 min walk distance. ${ }^{3}$ While these observations may be important in our understanding of the systemic consequences of IPAH, it remains to be determined if muscle wasting is caused by IPAH. Furthermore, similar changes in muscle morphology and strength are observed in other pulmonary diseases, suggesting a generalised process.

Peripheral muscle dysfunction is a major systemic consequence of COPD and has been well characterised. ${ }^{4}$ In COPD reasons for sarcopaenia are thought to be multifactorial, albeit with the effects of disuse accepted as the principle driver. This is largely supported by the morphological and functional reversal of changes seen following exercise training. The relative contributions of systemic inflammation, oxidative stress, tissue hypoxaemia, corticosteroids and malnutrition are unclear. Compared with matched controls, patients with COPD exhibit preferential loss of lower limb mass, strength and endurance. ${ }^{5}$ In particular, the emphysema phenotype is most associated with muscle loss; the carbon monoxide transfer coefficient $\left(\mathrm{K}_{\mathrm{CO}}\right)$ is a strong correlate of muscle mass. ${ }^{6}$ In general, histological and biochemical changes found within the quadriceps have favoured loss of endurance or aerobic muscle function. Quadriceps strength directly correlates to exercise capacity and peak oxygen uptake, and muscle cross-sectional area and strength have both been shown to be strong independent predictors of mortality. ${ }^{7}$ These data clearly place skeletal muscle dysfunction as being implicated in the clinical manifestations of COPD. Moreover, the impact of poorly efficient musculature may be compounded in COPD by competition for oxygen from socalled "respiratory steal".

In idiopathic pulmonary fibrosis (IPF) peripheral muscle weakness also contributes to exercise endurance. ${ }^{9}$ Quadriceps strength correlates with exercise capacity and is an independent predictor of peak oxygen consumption. Several other studies report peripheral muscle dysfunction in different systemic conditions, including chronic heart and renal failure. Patients with chronic heart failure demonstrate increased blood lactate levels and reduced peripheral oxygen consumption despite normal leg blood flow, suggesting that skeletal muscle dysfunction is responsible for this anaerobic metabolism. ${ }^{10}$ Uraemia in chronic kidney disease is associated with low protein stores, and the catabolic state observed in renal failure is thought to induce muscle atrophy. ${ }^{11}$ It is therefore highly plausible that skeletal muscle dysfunction results from factors common to many chronic diseases, rather than an intrinsic disease process. Indeed, recent developments in our understanding of the molecular mechanisms of muscle atrophy support this hypothesis.

Muscle mass is critically determined by the rate of protein synthesis versus protein degradation, and it is established that the predominant mechanism for muscle atrophy is accelerated proteolysis. Different atrophy stimuli (ranging from disuse, to glucocorticoids, to acidosis, to cachexia) activate a common transcriptional profile that induces protein breakdown, principally through activation of the UPS. ${ }^{12-14}$ Elegant studies in animal models have shown that these diverse atrophy-inducing stimuli initiate a common biological pathway, triggered by activation of Forkhead transcription factors (particularly FoxO1 and 3) ${ }^{15} 16$ and alterations in expression of a set of 
〜80 genes, termed "atrogenes", which include several components of the ubiquitin pathway. ${ }^{13} 1718$ In particular, two atrophy-specific ubiquitin E3 ligases, MuRF1 and atrogin-1 (MAFbx), are upregulated in catabolic states and are essential for rapid muscle wasting in animal models. ${ }^{19}$ Although the substrates and detailed molecular mechanisms of these muscle ligases remain to be determined, upregulation of this FoxO-mediated pathway has been observed in human disease. FoxO1, atrogin-1 and MuRF1 transcripts are upregulated in the quadriceps muscles of patients with COPD with peripheral muscle dysfunction when compared with controls, ${ }^{20}$ and complete diaphragmatic inactivity in ventilated brain-dead organ donors is associated with significant increases in muscle atrogin-1 and MuRF1 mRNA expression. ${ }^{21}$ However, not all studies demonstrate consistent findings. Diaphragmatic biopsies in patients with mild to moderate COPD show a significant increase in atrogin-1 transcripts but no change in MuRF1 expression. ${ }^{22}$ Changes in gene transcripts do not directly equate with protein levels, and with the paucity of data on MuRF1 and atrogin-1 protein expression it remains difficult to attribute particular conclusions to these human studies. It is therefore essential that future studies determine the detailed molecular mechanisms involved in muscle atrophy and, in particular, the temporal relationship of protein expression of muscle ubiquitin ligases to ubiquitinated protein species within atrophying muscle.

Whilst activation of the UPS is the major component of atrophy induction, many other cellular processes may potentially influence muscle mass and function. Factors such as corticosteroids and hormone changes may induce muscle wasting through distinct mechanisms such as inhibition of IGF-1 (insulin like growth factor-1), a growth factor that increases muscle mass by increasing protein synthesis and myogenesis, or by inducing myostatin, a secreted growth factor that negatively regulates muscle mass. Further studies are therefore essential to elucidate how these complex pathways are impacted by respiratory disease to induce muscle dysfunction, and examine their relative contribution to rapid muscle proteolysis compared with the UPS.

One hopes that understanding the detailed molecular biology of muscle atrophy will provide the basis for future drug targets and guide the impact of therapeutic strategies on muscle function. Moreover, as demonstrated in the study of Mainguy et al it is appropriate to explore the implications of these findings in respiratory diseases other than COPD as well as in different debilitating long-term conditions.

\section{Competing interests None.}

Provenance and peer review Commissioned; not externally peer reviewed.

Thorax 2010;65:97-98.

doi:10.1136/thx.2009.120824

\section{REFERENCES}

1. Mainguy V, Maltais F, Saey D, et al. Peripheral muscle dysfunction in idiopathic pulmonary arterial hypertension. Thorax 2010;65:113-17.

2. Meyer FJ, Lossnitzer D, Kristen AV, et al. Respiratory muscle dysfunction in idiopathic pulmonary arterial hypertension. Eur Respir $\mathrm{J}$ 2005;25:125-30.

3. Bauer R, Dehnert C, Schoene P, et al. Skeletal muscle dysfunction in patients with idiopathic pulmonary arterial hypertension. Respir Med 2007:101:2366-9.

4. American Thoracic Society and European Respiratory Society. Skeletal muscle dysfunction in chronic obstructive pulmonary disease. A statement of the American Thoracic Society and European Respiratory Society. Am J Respir Crit Care Med 1999;159:S1-40.

5. Bernard S, LeBlanc P, Whittom F, et al. Peripheral muscle weakness in patients with chronic obstructive pulmonary disease. Am J Respir Crit Care Med 1998; 158:629-34

6. Engelen MP, Schols AM, Baken WC, et al. Nutritional depletion in relation to respiratory and peripheral skeletal muscle function in out-patients with COPD. Eur Respir J 1994;7:1793-7.
7. Swallow EB, Reyes D, Hopkinson NS, et al. Quadriceps strength predicts mortality in patients with moderate to severe chronic obstructive pulmonary disease. Thorax 2007;62:115-20.

8. Chiappa GR, Queiroga F Jr, Meda E, et al. Heliox improves oxygen delivery and utilization during dynamic exercise in patients with chronic obstructive pulmonary disease. Am J Respir Crit Care Med 2009;179:1004-10.

9. Nishiyama $\mathbf{0}$, Taniguchi $\mathrm{H}$, Kondoh $\mathrm{Y}$, et al. Quadriceps weakness is related to exercise capacity in idiopathic pulmonary fibrosis. Chest 2005: 127:2028-33

10. Duscha BD, Schulze PC, Robbins JL, et al. Implications of chronic heart failure on peripheral vasculature and skeletal muscle before and after exercise training. Heart Fail Rev 2008;13:21-37.

11. Du J, Hu Z, Mitch WE. Molecular mechanisms activating muscle protein degradation in chronic kidney disease and other catabolic conditions. Eur $\mathrm{J}$ Clin Invest 2005;35:157-63.

12. Jagoe RT, Goldberg AL. What do we really know about the ubiquitin-proteasome pathway in muscle atrophy? Curr Opin Clin Nutr Metab Care 2001;4:183-90.

13. Lecker SH, Jagoe RT, Gilbert A, et al. Multiple types of skeletal muscle atrophy involve a common program of changes in gene expression. FASEB J 2004; 18:39-51.

14. Mitch WE, Goldberg AL. Mechanisms of muscle wasting. The role of the ubiquitin-proteasome pathway. N Engl J Med 1996;335:1897-905.

15. Zhao J, Brault JJ, Schild A, et al. Fox03 coordinately activates protein degradation by the autophagic/ lysosomal and proteasomal pathways in atrophying muscle cells. Cell Metab 2007:6:472-83.

16. Sandri M, Sandri C, Gilbert A, et al. Foxo transcription factors induce the atrophy-related ubiquitin ligase atrogin-1 and cause skeletal muscle atrophy. Cell 2004;117:399-412.

17. Gomes MD, Lecker SH, Jagoe RT, et al. Atrogin-1, a muscle-specific F-box protein highly expressed during muscle atrophy. Proc Natl Acad Sci USA 2001; 98:14440-5

18. Jagoe RT, Lecker SH, Gomes M, et al. Patterns of gene expression in atrophying skeletal muscles: response to food deprivation. FASEB J 2002;16:1697-712.

19. Bodine SC, Latres E, Baumhueter S, et al. Identification of ubiquitin ligases required for skeletal muscle atrophy. Science 2001;294:1704-8.

20. Doucet M, Russell AP, Leger B, et al. Muscle atrophy and hypertrophy signaling in patients with chronic obstructive pulmonary disease. Am J Respir Crit Care Med 2007;176:261-9

21. Levine S, Nguyen T, Taylor N, et al. Rapid disuse atrophy of diaphragm fibers in mechanically ventilated humans. N Engl J Med 2008;358:1327-35.

22. Ottenheijm CA, Heunks LM, Li YP, et al. Activation of the ubiquitin-proteasome pathway in the diaphragm in chronic obstructive pulmonary disease. Am J Respir Crit Care Med 2006:174:997-1002. 